\title{
A novel ionic liquid-supported Schiff base ligand applied in the Pd- catalyzed Suzuki-Miyaura coupling reaction
}

\author{
Bin Li, Yi-Qun Li* and Jia Zheng \\ Department of Chemistry, Jinan University, Guangzhou, 510632, China \\ E-mail: tlyq@jnu.edu.cn
}

\begin{abstract}
A series of novel ionic liquid-supported Schiff bases have been prepared from the condensation of aromatic aldehydes with the ionic liquid 1-(2-aminoethyl)-3-methylimidazolium hexafluorophosphate. The as-prepared ionic liquid-supported salicyladehyde Schiff base L3 was further investigated as a ligand for the Pd-catalyzed Suzuki-Miyaura coupling reaction under air in ethanol solution, giving biaryls in good to excellent yields. The $\mathrm{PdCl}_{2} /$ ligand $\mathbf{L} 3$ catalytic system could be reused at least 5 times without losing its activity.
\end{abstract}

Keywords: Ionic liquid, Schiff base, palladium chloride, Suzuki-Miyaura reaction, ligand

\section{Introduction}

Transition-metal-catalyzed cross-coupling is a versatile and highly useful transformation, which yields a variety of organic compounds. In particular, the Suzuki-Miyaura cross-coupling reaction, which is the palladium-catalyzed cross coupling reaction of aryl halides with organoboronic compounds, represents one of the most important methods of forming $\mathrm{sp}^{2}-\mathrm{sp}^{2}$ carbon-carbon bonds in synthetic chemistry, as well as in industrial applications. ${ }^{1-4}$ In the past few years, many attempts have been made to develop effective palladium complexes, which can act as highly active catalysts for this homogeneous reaction. Among them, various forms of ligands such as phosphorous ligands, ${ }^{5-8} \mathrm{P}$, O-based ligands, ${ }^{9-10}$ bis(thiourea) ligands, ${ }^{11}$ thiosemicarbazone, ${ }^{12}$ etc. were used to stabilize the catalytic palladium species. Although these catalysts often have higher activities in such transformations, however, most of them are expensive, not easy available, require air-free handling to prevent the oxidation, and exist the main drawback of these catalysts is the necessity of their separation from the reaction mixture at the end of the reaction.

Recently, the use of ionic liquids (ILs) has received more attention as ecofriendly, reusable

and alternative reaction media in organic synthesis because of their unique properties. ${ }^{13-15}$ The capacity of ILs to solvate both polar and nonpolar species allows the dissolution of a wide range 
of organic, inorganic, and organometallic compounds favoring the formation of a homogeneous catalytic system. However, many palladium (II)-catalyzed coupling reactions are carried out in ILs in the presence of bulky phosphine ligands, ${ }^{16-18}$ which are sensitive to both oxygen and moisture, and lead to catalyst decomposition. Thus, the development of phosphine-free recyclable catalysts is of great importance. ${ }^{19-22}$ Most recently, much more attention has been focused on the synthesis of functionalized ionic liquids (FILs), via incorporation of additional functional groups such as amine, amide, nitrile, ether, alcohol, acid, urea or thiourea, etc. as a part of the cation and/or anion for task specific purposes. The FILs have shown great promise not only as alternative green solvents, but also as reagents or catalysts in organic transformations. ${ }^{23-}$ 26

We have been interested in the use of functionalized ionic liquid-supported Schiff base, since Schiff base palladium(II) compounds are excellent candidates as N, O- bidentate ligands in catalysis owing to their desirable coordinating ability with palladium. In this paper, we wish to report the use of ionic liquid-supported Schiff base as the palladium (II) ligand to demonstrate their suitability in palladium-catalyzed Suzuki-Miyaura cross-coupling reactions.

\section{Results and Discussion}

The ligands L1-L6 of ionic liquid supported Schiff base were readily prepared by the condensation of aromatic aldehydes with the functionalized ionic liquid 1-(2-aminoethyl)-3methylimidazolium hexafluorophosphate (Scheme 1).

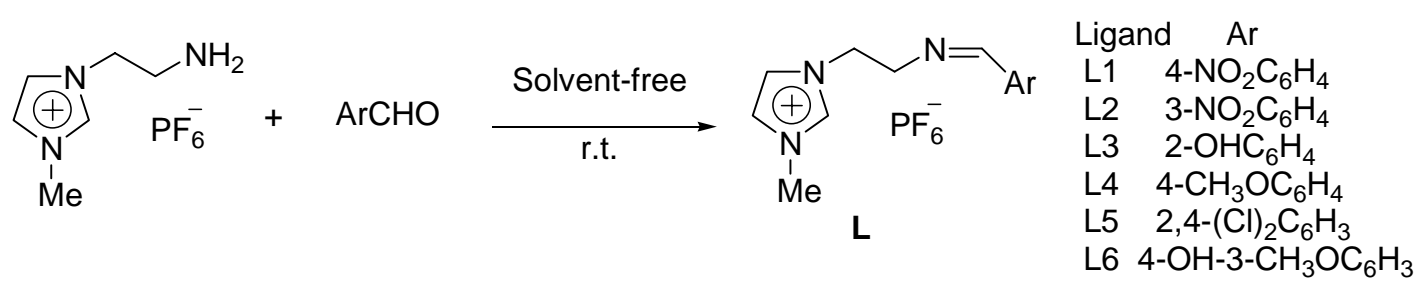

Scheme 1. Synthesis of the ionic liquid supported Schiff bases.

To evaluate the activity of the catalyst formed in situ from ligand L1-L6 and $\mathrm{PdCl}_{2}$ in Suzuki-Miyaura reaction, the coupling reaction of 4-iodoanisole with phenylboronic acid has been chosen as a model reaction (Table 1). It can be seen that when using $\mathbf{L} 3$ as ligand, the reaction proceeded much faster and the product obtained with higher yields than other ligands (Table 1, entry 3 ). 
Table 1. Influence of ligands $\mathbf{L}$ on the palladium-catalyzed Suzuki-Miyaura coupling of 4indoanisole with phenylboronic acid ${ }^{\mathrm{a}}$

\begin{tabular}{cccc}
\hline Entry & Ligand & Time $(\mathrm{h})$ & ${\text { Yield }(\%)^{\mathrm{b}}}^{\mathrm{b}}$ \\
\hline 1 & L1 & 1 & 87 \\
2 & L2 & 1 & 88 \\
3 & L3 & 0.5 & 98 \\
4 & L4 & 0.5 & 87 \\
5 & L5 & 1.5 & 92 \\
6 & L6 & 0.5 & 87 \\
\hline
\end{tabular}

${ }^{\mathrm{a}}$ Reaction conditions: 4-indoanisole $(1.0 \mathrm{mmol})$, phenylboronic acid $(1.2 \mathrm{mmol}), \mathrm{PdCl}_{2}$ $(0.5 \mathrm{~mol} \%)$, Ligand $(0.5 \mathrm{~mol} \%), \mathrm{C}_{2} \mathrm{H}_{5} \mathrm{OH}(10 \mathrm{~mL}), \mathrm{K}_{2} \mathrm{CO}_{3}(2 \mathrm{mmol})$, at reflux under air.

${ }^{\mathrm{b}}$ Isolated yields

Encouraged by this result, we further investigated the effect of base, solvent and temperatures on the Suzuki-Miyaura reaction when using $\mathbf{L 3}$ as ligand under the same condition mentioned above. The results were listed in Table 2. Using $\mathrm{K}_{2} \mathrm{CO}_{3}$ as a base, the reaction gave good yield of the desired product (Table 2, entry 1). Several commonly used solvents were

Table 2. Effect of bases, solvents, and temperatures on the Suzuki-Miyaura coupling reaction of 4-indoanisole and phenylboronic acid ${ }^{\mathrm{a}}$

\begin{tabular}{|c|c|c|c|c|c|}
\hline Entry & Base & Solvent & $\operatorname{Temp}\left({ }^{\circ} \mathrm{C}\right)$ & Time(h) & Yield $(\%)^{b}$ \\
\hline 1 & $\mathrm{~K}_{2} \mathrm{CO}_{3}$ & EtOH & 78 & 0.5 & 98 \\
\hline 2 & $\mathrm{Na}_{2} \mathrm{CO}_{3}$ & $\mathrm{EtOH}$ & 78 & 1 & 46 \\
\hline 3 & $\mathrm{KOH}$ & $\mathrm{EtOH}$ & 78 & 1 & 75 \\
\hline 4 & $\mathrm{NaOH}$ & $\mathrm{EtOH}$ & 78 & 0.5 & 87 \\
\hline 5 & $\mathrm{NaHCO}_{3}$ & $\mathrm{EtOH}$ & 78 & 5 & 33 \\
\hline 6 & $\mathrm{CaO}$ & $\mathrm{EtOH}$ & 78 & 5 & 27 \\
\hline 7 & $\mathrm{CsCO}_{3}$ & $\mathrm{EtOH}$ & 78 & 0.5 & 93 \\
\hline 8 & $\mathrm{Na}_{3} \mathrm{PO}_{4} \cdot 12 \mathrm{H}_{2} \mathrm{O}$ & EtOH & 78 & 0.5 & 65 \\
\hline 9 & $\mathrm{~K}_{2} \mathrm{CO}_{3}$ & $\mathrm{H}_{2} \mathrm{O}$ & 78 & 1 & 38 \\
\hline 10 & $\mathrm{~K}_{2} \mathrm{CO}_{3}$ & $\mathrm{CH}_{3} \mathrm{CN}$ & 78 & 5.5 & 60 \\
\hline 11 & $\mathrm{~K}_{2} \mathrm{CO}_{3}$ & DMF & 78 & 1 & 87 \\
\hline 12 & $\mathrm{~K}_{2} \mathrm{CO}_{3}$ & cyclohexane & 78 & 5 & $\mathrm{NR}^{\mathrm{c}}$ \\
\hline 13 & $\mathrm{~K}_{2} \mathrm{CO}_{3}$ & $\mathrm{EtOH}$ & 50 & 1 & 88 \\
\hline
\end{tabular}

a Reaction conditions: 4-indoanisole (1.0 mmol), phenylboronic acid (1.2 $\mathrm{mmol}), \mathrm{PdCl}_{2}$ $(0.5 \mathrm{~mol} \%), \mathbf{L 3}(0.5 \mathrm{~mol} \%)$, solvent $(10 \mathrm{~mL})$, base $(2 \mathrm{mmol})$, under air.

${ }^{\mathrm{b}}$ Isolated yields

${ }^{\mathrm{c}}$ No reaction 
tested. The nonpolar solvent cyclohexane and protic solvent $\mathrm{H}_{2} \mathrm{O}$ was found not to be effective (Table 2, entries 9 and 12), and polar solvents, such as EtOH, DMF and $\mathrm{CH}_{3} \mathrm{CN}$, were preferred for this reaction and the EtOH (Table 2, entries 1-8 and 13) was the best choice. The reaction temperature has dramatic effect on the reaction, when the reaction carried out at $50^{\circ} \mathrm{C}$, the yield decreased to $88 \%$ from $98 \%$ (Table 2 , entry 13 ). In addition, the reactions were performed in air and without degassing the solvent prior to use.

After having established the optimized coupling reaction conditions, the scope of the reaction and efficiencies of the ligand L3 were evaluated by investigating the coupling of various substituted aryl halides and substituted aryl boronic acid (Scheme 2).

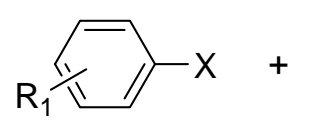

1

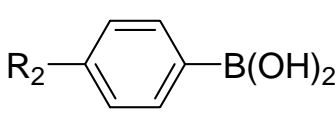

2

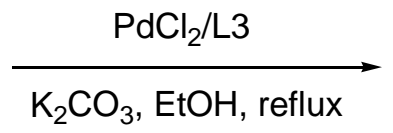

$\mathrm{K}_{2} \mathrm{CO}_{3}, \mathrm{EtOH}$, reflux

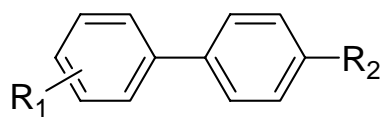

3

Scheme 2. Suzuki-Miyaura reaction catalyzed by $\mathrm{PdCl}_{2} / \mathrm{L} 3$ system.

The results were shown in Table 3. As shown in Table 3, the aryl iodides produced good yields in shortest times irrespective of the substituents on aryl halides and aryl boronic acid (Table 3, entries 1-11). Aryl bromides with different substituents also gave good yields in the coupling reaction except $O$-nitrobromobenzene due to the steric effect (Table 3, entries 12-19). However, aryl chloride gave a poor yield under the same optimized reaction conditions (Table 3 , entry 20).

Table 3. $\mathrm{PdCl}_{2} /$ Ligand $\mathrm{L} 3$ catalyzed coupling reaction of aryl halides with aryl boronic acids ${ }^{\mathrm{a}}$

\begin{tabular}{ccccccc}
\hline Entry & $\mathrm{X}$ & $\mathrm{R}_{1}$ & $\mathrm{R}_{2}$ & Product & Time(h) & Yield(\%) $^{\mathrm{b}}$ \\
\hline 1 & $\mathrm{I}$ & $\mathrm{H}$ & $\mathrm{H}$ & 1,1'-Biphenyl 3a & 0.5 & 92 \\
\hline 2 & $\mathrm{I}$ & $\mathrm{H}$ & $\mathrm{Cl}$ & 4-Chlorobiphenyl 3b & 0.5 & 94 \\
\hline 3 & $\mathrm{I}$ & $\mathrm{H}$ & $\mathrm{F}$ & 4-Fluorobiphenyl 3c & 0.5 & 92 \\
\hline 4 & $\mathrm{I}$ & $\mathrm{H}$ & $\mathrm{CH}_{3}$ & 4-Methylbiphenyl 3d & 0.5 & 93 \\
\hline 5 & $\mathrm{I}$ & $\mathrm{H}$ & $\mathrm{OCH}_{3}$ & 4-Methoxybiphenyl 3e & 0.5 & 98 \\
\hline 6 & $\mathrm{I}$ & 4-OCH & $\mathrm{H}$ & 4-Methoxybiphenyl 3e & 0.5 & 98 \\
\hline 7 & $\mathrm{I}$ & $4-\mathrm{OCH}_{3}$ & $\mathrm{Cl}$ & 4-Chloro-4'-methoxybiphenyl 3f & 0.5 & 84 \\
\hline 8 & $\mathrm{I}$ & 4- $\mathrm{OCH}_{3}$ & $\mathrm{~F}$ & 4-Fluoro-4'-methoxybiphenyl 3g & 0.5 & 87 \\
\hline 9 & $\mathrm{I}$ & $4-\mathrm{OCH}_{3}$ & $\mathrm{CH}_{3}$ & 4-Methoxy-4'-methylbiphenyl 3h & 0.5 & 96 \\
\hline 10 & $\mathrm{I}$ & 4-OCH & $\mathrm{OCH}_{3}$ & 4,4'-Dimethoxybiphenyl 3i & 0.5 & 84 \\
\hline 11 & $\mathrm{I}$ & 4- $\mathrm{COOH}$ & $\mathrm{H}$ & 4-Biphenylylcarboxylic acid 3j & 1 & 95 \\
\hline 12 & $\mathrm{Br}$ & $\mathrm{H}$ & $\mathrm{Cl}$ & 4-Chlorobiphenyl 3b & 0.5 & 88 \\
\hline
\end{tabular}


Table 3. Continued

\begin{tabular}{ccccccc}
\hline Entry & $\mathrm{X}$ & $\mathrm{R} 1$ & $\mathrm{R} 2$ & Product & Time(h) & Yield(\%)b \\
\hline 13 & $\mathrm{Br}$ & $\mathrm{H}$ & $\mathrm{OCH}_{3}$ & 4-Methoxybiphenyl 3e & 0.5 & 89 \\
\hline 14 & $\mathrm{Br}$ & 4- $\mathrm{NO}_{2}$ & $\mathrm{H}$ & 4-Nitrobiphenyl 3k & 1 & 95 \\
\hline 15 & $\mathrm{Br}$ & 3- $\mathrm{NO}_{2}$ & $\mathrm{H}$ & 3-Nitrobiphenyl 3l & 6 & 86 \\
\hline 16 & $\mathrm{Br}$ & 4-OH & $\mathrm{H}$ & 4-Hydroxybiphenyl 3m & 1.5 & 84 \\
\hline 17 & $\mathrm{Br}$ & 4- $\mathrm{COOCH}_{3}$ & $\mathrm{H}$ & 4-Methoxycarbonylbiphenyl 3n & 3 & 86 \\
\hline 18 & $\mathrm{Br}$ & 4- $\mathrm{OCH}_{3}$ & $\mathrm{H}$ & 4-Methoxybiphenyl 3o & 1.5 & 94 \\
\hline 19 & $\mathrm{Br}$ & 2- $\mathrm{NO}_{2}$ & $\mathrm{H}$ & 2-Nitrobiphenyl 3p & 5 & $\mathrm{NR}^{\mathrm{c}}$ \\
\hline 20 & $\mathrm{Cl}$ & $4-\mathrm{NO}_{2}$ & $\mathrm{H}$ & 4-Nitrobiphenyl 3k & 24 & $44^{\mathrm{d}}$ \\
\hline
\end{tabular}

${ }^{\mathrm{a}}$ Reaction conditions: aryl halides $(1.0 \mathrm{mmol})$, aryl boronic acids $(1.2 \mathrm{mmol}), \mathrm{PdCl}_{2}(0.5 \mathrm{~mol} \%)$, Ligand $\mathbf{L 3}(0.5 \mathrm{~mol} \%), \mathrm{C}_{2} \mathrm{H}_{5} \mathrm{OH}(10 \mathrm{~mL}), \mathrm{K}_{2} \mathrm{CO}_{3}(2 \mathrm{mmol})$, at reflux under air.

${ }^{\mathrm{b}}$ Isolated yields.

${ }^{\mathrm{c}}$ No reaction.

$\mathrm{d}_{2}$ mol$\% \mathrm{PdCl}_{2}, 2$ mol\% Ligand $\mathbf{L} 3$ used.

One of the main aims of our study was to investigate the reuse and recycling the catalyst. Finally, we explored the reusability of the $\mathrm{PdCl}_{2}$ /ligand $\mathbf{L} 3$ catalytic system using the reaction of 4-iodoanisole and phenylboronic acid as the model reaction. After the completion of the reaction, the reaction mixture was diluted with $\mathrm{Et}_{2} \mathrm{O}$ and decanted, the remained catalyst was washed with $\mathrm{Et}_{2} \mathrm{O}$ for several times and reused directly under the similar conditions mentioned above. The results listed in Table 4 and showed the catalytic system could be reused up to 5 runs while retaining the catalytic activity.

Table 4. Reuse of catalytic system on the Suzuki-Miyaura coupling reaction of 4-indoanisole and phenylboronic acid ${ }^{\mathrm{a}}$

\begin{tabular}{cccccc}
\hline Run & 1 & 2 & 3 & 4 & 5 \\
\hline${\text { Yield }(\%)^{\mathrm{b}}}^{\mathrm{n}}$ & 98 & 95 & 96 & 94 & 93 \\
\hline
\end{tabular}

${ }^{a}$ Reaction conditions: 4-iodoanisole $(2.0 \mathrm{mmol})$, phenylboronic acid $(2.4 \mathrm{mmol}), \mathrm{PdCl}_{2}(1$ mol\%), Ligand $\mathrm{L} 3(1 \mathrm{~mol} \%), \mathrm{C}_{2} \mathrm{H}_{5} \mathrm{OH}(20 \mathrm{~mL}), \mathrm{K}_{2} \mathrm{CO}_{3}(4.0 \mathrm{mmol})$, at reflux under air.

${ }^{\mathrm{b}}$ Isolated yields.

\section{Conclusions}

We have demonstrated the $\mathrm{PdCl}_{2} /$ ligand $\mathbf{L} 3$ catalyst was proved to be highly efficient in Suzukimiyaura reactions of aryl halides and aryl boronic acids. Furthermore, the $\mathrm{PdCl}_{2} /$ ligand $\mathbf{L} 3$ 
catalyst can be easily separated and recovered from the reaction mixture by filtration and reused for up to 5 runs without noticeable losing activities. The utility of the $\mathrm{PdCl}_{2}$ /ligand $\mathbf{L}$ catalysts in other coupling reactions such as Heck and Sonogashira reactions is being explored in our laboratory.

\section{Experimental Section}

General. All products were characterized by comparison of their spectra and physical data with authentic samples. Melting points were measured on an Electrothemal X6 microscopy digital melting point apparatus. IR spectra were recorded on a Bruker Equinox 55 spectrometer using KBr pellets. ${ }^{1} \mathrm{H}$ NMR spectra were recorded on a Bruker AVANCE 300 (300 MHz) instrument with the TMS at d $0.00 \mathrm{ppm}$ as an internal standard. Elemental analyses were carried out on a PE EA2400 CHN analyzer. All chemicals were reagent grade and used as purchased.

Synthesis of the ionic liquid supported Schiff bases. ${ }^{27}$ A mixture of the ionic liquid 1-(2aminoethyl)-3-methylimidazolium hexafluorophosphate $(1.028 \mathrm{~g}, 4.0 \mathrm{mmol})$ and aromatic aldehydes $(3.0 \mathrm{mmol})$ was stirred for $4 \mathrm{~h}$ at room temperature under solvent-free conditions. After completion of the reaction, ethanol $(30 \mathrm{~mL})$ was added to the reaction mixture, the solid product formed was filtered off and washed with cold ethanol. The crude product was purified by recrystallization from ethanol/ethyl acetate $(3: 1 \mathrm{v} / \mathrm{v})$. The structure for $\mathbf{L} 3$ was characterized by $\mathrm{X}$-ray single crystal diffraction (CCDC 712469).

1-[2-(4-Nitrobenzylideneamino)ethyl]-3-methyl-3H-imidazol-3-ium hexafluorophosphate (L1). White solid; mp 180-182 ${ }^{\circ} \mathrm{C}$. IR (KBr): $1641(\mathrm{C}=\mathrm{N}), 829 \mathrm{~cm}^{-1}(\mathrm{P}-\mathrm{F}) .{ }^{1} \mathrm{H}$ NMR $(300 \mathrm{MHz}$, DMSO-d $\left.)_{6}\right): \delta 3.83\left(\mathrm{~s}, 3 \mathrm{H}, \mathrm{CH}_{3}\right), 4.01\left(\mathrm{t}, J=5.33 \mathrm{~Hz}, 2 \mathrm{H}, \mathrm{CH}_{2}\right), 4.52\left(\mathrm{t}, J=5.27 \mathrm{~Hz}, 2 \mathrm{H}, \mathrm{CH}_{2}\right)$, 7.67 (s, 1H, NCH), 7.76 (s, 1H, NCH), 7.97, 7.99 (AA', 2H, Ar-H), 8.28, 8.31 (BB', 2H, Ar-H), $8.48(\mathrm{~s}, 1 \mathrm{H}, \mathrm{N}=\mathrm{CH}), 9.12(\mathrm{~s}, 1 \mathrm{H}, \mathrm{N}(\mathrm{H}) \mathrm{CN})$. Anal. calcd. for $\mathrm{C}_{13} \mathrm{H}_{15} \mathrm{~F}_{6} \mathrm{~N}_{4} \mathrm{O}_{2} \mathrm{P}: \mathrm{C}, 38.63 ; \mathrm{H}, 3.74$; N, 13.86. Found: C, 38.48; H, 3.58; N, 13.62 .

1-[2-(3-Nitrobenzylideneamino)ethyl]-3-methyl-3H-imidazol-3-ium hexafluorophosphate (L2). White solid; mp 133-135 ${ }^{\circ} \mathrm{C}$. IR (KBr): $1645(\mathrm{C}=\mathrm{N}), 838 \mathrm{~cm}^{-1}$ (P-F). ${ }^{1} \mathrm{H}$ NMR (300 MHz, DMSO-d $)_{6}$ : $\delta 3.09\left(\mathrm{~s}, 3 \mathrm{H}, \mathrm{CH}_{3}\right), 3.25\left(\mathrm{t}, J=5.20 \mathrm{~Hz}, 2 \mathrm{H}, \mathrm{CH}_{2}\right), 3.78\left(\mathrm{t}, J=5.32 \mathrm{~Hz}, 2 \mathrm{H}, \mathrm{CH}_{2}\right.$ ), $6.73(\mathrm{~s}, 1 \mathrm{H}, \mathrm{NCH}), 6.85(\mathrm{~s}, 1 \mathrm{H}, \mathrm{NCH}), 6.88-7.62(\mathrm{~m}, 4 \mathrm{H}, \mathrm{Ar}-\mathrm{H}), 7.78(\mathrm{~s}, 1 \mathrm{H}, \mathrm{N}=\mathrm{CH})$. Anal. calcd. for $\mathrm{C}_{13} \mathrm{H}_{15} \mathrm{~F}_{6} \mathrm{~N}_{4} \mathrm{O}_{2} \mathrm{P}: \mathrm{C}, 38.63 ; \mathrm{H}, 3.74 ; \mathrm{N}, 13.86$. Found: $\mathrm{C}, 38.05 ; \mathrm{H}, 3.38 ; \mathrm{N}, 13.62$.

1-[2-(2-Hydroxybenzylideneamino)ethyl]-3-methyl-3H-imidazol-3-ium hexafluorophosphate (L3). Yellow solid; mp 132-134 ${ }^{\circ} \mathrm{C}$. IR (KBr): $3422(\mathrm{O}-\mathrm{H}), 1640(\mathrm{C}=\mathrm{N}), 837 \mathrm{~cm}^{-1}(\mathrm{P}-\mathrm{F}) .{ }^{1} \mathrm{H}$ NMR (300 MHz, DMSO-d $\left.)_{6}\right): \delta 3.82\left(\mathrm{~s}, 3 \mathrm{H}, \mathrm{CH}_{3}\right), 3.99\left(\mathrm{t}, J=5.49 \mathrm{~Hz}, 2 \mathrm{H}, \mathrm{CH}_{2}\right), 4.52(\mathrm{t}, J=$ $\left.5.48 \mathrm{~Hz}, 2 \mathrm{H}, \mathrm{CH}_{2}\right), 6.85-7.42(\mathrm{~m}, 4 \mathrm{H}, \mathrm{Ar}-\mathrm{H}), 7.67$ (s, 1H, NCH), 7.73 (s, 1H, NCH), 8.50 (s, 1H, $\mathrm{N}=\mathrm{CH}), 9.12(\mathrm{~s}, 1 \mathrm{H}, \mathrm{OH})$. Anal. calcd. for $\mathrm{C}_{13} \mathrm{H}_{16} \mathrm{~F}_{6} \mathrm{~N}_{3} \mathrm{OP}: \mathrm{C}, 41.61 ; \mathrm{H}, 4.29 ; \mathrm{N}, 11.20$. Found: C, 40.81; H, 4.19; N, 10.99 . 
1-[2-(4-Methoxybenzylideneamino)ethyl]-3-methyl-3H-imidazol-3-ium hexafluoro-phosphate (L4). Yellow solid; mp 122-124 ${ }^{\circ} \mathrm{C}$. IR (KBr): $1644(\mathrm{C}=\mathrm{N}), 847 \mathrm{~cm}^{-1}(\mathrm{P}-\mathrm{F}) .{ }^{1} \mathrm{H}$ NMR (300 MHz, DMSO-d $\left.)_{6}\right): \delta .01\left(\mathrm{~s}, 3 \mathrm{H}, \mathrm{CH}_{3}\right), 3.06\left(\mathrm{~s}, 3 \mathrm{H}, \mathrm{CH}_{3}\right), 3.14\left(\mathrm{t}, J=5.16 \mathrm{~Hz}, 2 \mathrm{H}, \mathrm{CH}_{2}\right), 3.70(\mathrm{t}, J=$ $\left.5.29 \mathrm{~Hz}, 2 \mathrm{H}, \mathrm{CH}_{2}\right), 6.13,6.16\left(\mathrm{AA}^{\prime}, 2 \mathrm{H}, \mathrm{Ar}-\mathrm{H}\right), 6.70(\mathrm{~s}, 1 \mathrm{H}, \mathrm{NCH}), 6.79(\mathrm{~s}, 1 \mathrm{H}, \mathrm{NCH}), 6.83$, $6.86\left(\mathrm{BB}^{\prime}, 2 \mathrm{H}, \mathrm{Ar}-\mathrm{H}\right), 7.37(\mathrm{~s}, 1 \mathrm{H}, \mathrm{N}=\mathrm{CH})$. Anal. calcd. for $\mathrm{C}_{14} \mathrm{H}_{18} \mathrm{~F}_{6} \mathrm{~N}_{3} \mathrm{OP}: \mathrm{C}, 43.20 ; \mathrm{H}, 4.66 ; \mathrm{N}$, 10.79. Found: C, 42.29; H, 4.61; N, 10.55.

1-[2-(2,4-Dichlorobenzylideneamino)ethyl]-3-methyl-3H-imidazol-3-ium hexafluoro- phosphate (L5). White solid; mp 129-131 ${ }^{\circ} \mathrm{C}$. IR ( $\left.\mathrm{KBr}\right): 1638(\mathrm{C}=\mathrm{N}), 835 \mathrm{~cm}^{-1}(\mathrm{P}-\mathrm{F}) .{ }^{1} \mathrm{H}$ NMR $(300 \mathrm{MHz}$, DMSO-d $\left.)_{6}\right): \delta .08\left(\mathrm{~s}, 3 \mathrm{H}, \mathrm{CH}_{3}\right), 3.23\left(\mathrm{t}, J=5.43 \mathrm{~Hz}, 2 \mathrm{H}, \mathrm{CH}_{2}\right), 3.75\left(\mathrm{t}, J=5.43 \mathrm{~Hz}, 2 \mathrm{H}, \mathrm{CH}_{2}\right)$, $6.58(\mathrm{~d}, J=8.61 \mathrm{~Hz}, 1 \mathrm{H}, \mathrm{Ar}-\mathrm{H}), 6.71(\mathrm{~s}, 1 \mathrm{H}, \mathrm{NCH}), 6.73(\mathrm{~s}, 1 \mathrm{H}, \mathrm{NCH}), 6.84(\mathrm{~s}, 1 \mathrm{H}, \mathrm{Ar}-\mathrm{H}), 7.17$ $(\mathrm{d}, J=8.46 \mathrm{~Hz}, 1 \mathrm{H}, \mathrm{Ar}-\mathrm{H}), 7.84(\mathrm{~s}, 1 \mathrm{H}, \mathrm{N}=\mathrm{CH})$. Anal. calcd. for $\mathrm{C}_{13} \mathrm{H}_{14} \mathrm{Cl}_{2} \mathrm{~F}_{6} \mathrm{~N}_{3} \mathrm{P}: \mathrm{C}, 36.47 ; \mathrm{H}$, $3.30 ; \mathrm{N}, 9.81$. Found: C, 36.41; H, 3.04; N, 9.71.

1-[2-(4-Hydroxy-3-methoxybenzylideneamino)ethyl]-3-methyl-3H-imidazol-3-ium hexafluorophosphate (L6). Yellow solid; mp 178-180 ${ }^{\circ} \mathrm{C}$. IR (KBr): $1643(\mathrm{C}=\mathrm{N}), 847 \mathrm{~cm}^{-1}$ (P-F). ${ }^{1} \mathrm{H}$ NMR $(300 \mathrm{MHz}$, DMSO-d $\left.)_{6}\right): \delta 3.06\left(\mathrm{~s}, 3 \mathrm{H}, \mathrm{CH}_{3}\right), 3.07\left(\mathrm{~s}, 3 \mathrm{H}, \mathrm{CH}_{3}\right), 3.15\left(\mathrm{t}, J=5.59 \mathrm{~Hz}, 2 \mathrm{H}, \mathrm{CH}_{2}\right), 3.71(\mathrm{t}, J=$ $\left.5.49 \mathrm{~Hz}, 2 \mathrm{H}, \mathrm{CH}_{2}\right), 5.98-6.31$ (m, 3H, Ar-H), 6.70 (s, 1H, NCH), 6.80 (s, 1H, NCH), 7.30 (s, 1H, $\mathrm{N}=\mathrm{CH}$ ). Anal. calcd. for $\mathrm{C}_{14} \mathrm{H}_{18} \mathrm{~F}_{6} \mathrm{~N}_{3} \mathrm{O}_{2} \mathrm{P}: \mathrm{C}, 41.49 ; \mathrm{H}, 4.48 ; \mathrm{N}, 10.37$. Found: $\mathrm{C}, 40.81 ; \mathrm{H}, 4.47$; $\mathrm{N}, 10.20$.

\section{General procedure for Suzuki-Miyaura reaction}

A mixture of aryl halide (1.0 mmol), arylboronic acid (1.2 mmol), L3 (0.005 mmol), $\mathrm{PdCl}_{2}$ $(0.005 \mathrm{mmol}), \mathrm{K}_{2} \mathrm{CO}_{3}(2.0 \mathrm{mmol})$, and $\mathrm{EtOH}(10.0 \mathrm{~mL})$ was added to a flask and stirred under reflux. After the reaction was judged to be complete by TLC analysis, the reaction mixture was cooled to room temperature, and to the solution was added $\mathrm{Et}_{2} \mathrm{O}(20 \mathrm{~mL})$, the $\mathrm{PdCl}_{2} / \mathbf{L} 3$ catalyst was remained in the flask bottom and was washed with $\mathrm{Et}_{2} \mathrm{OH}(3 \times 10 \mathrm{~mL})$. The organic phase was evaporated and the residue was purified by recrystallization. All of the products 3a-3k are known and the data are found to be identical with those that reported in literature elsewhere.

\section{Acknowledgements}

We are grateful to the National Natural Science Foundation of China (No. 20672046) and the Guangdong Natural Science Foundation (No. 8151063201000016) for financial support.

\section{References}

1. Miyaura, N.; Suzuki, A. Chem. Rev. 1995, 95, 2457.

2. Suzuki, A. J. Organomet. Chem. 1999, 576, 147.

3. Özdemir, I.; Demir, S.; Çetinkaya, B. Arkivoc 2007, (xiii), 71. 
4. Littke, A. F.; Fu, G. C. Angew. Chem. Int. Ed. 2002, 41, 4176.

5. Littke, A. F.; Fu, G. C. Angew. Chem. Int. Ed. 1998, 37, 3387.

6. Zapf, A.; Ehrentraut, A.; Beller, M. Angew. Chem. Int. Ed. 2000, 39, 4153.

7. Walker, S. D.; Barder, T. E.; Martinelli, J. R.; Buchwald, S. L. Angew. Chem. Int. Ed. 2004, 43, 1871.

8. Wolf, C.; Ekoue-Kovi, K. Eur. J. Org. Chem. 2006, 1917.

9. Dai, W. M.; Zhang, Y. Tetrahedron Lett. 2005, 46, 1377.

10. Dai, W. M.; Li, Y.; Zhang, Y.; Yue, C.; Wu, J. Chem. Eur. J. 2008, 14, 5538.

11. Chen, W.; Li, R.; Han, B.; Li, B. J.; Chen, Y. C.; Ding, L. S.; Yang, D. Eur. J. Org. Chem. 2006, 1177.

12. Kovala-Demertzi, D.; Kourkoumelis, N.; Derlat, K.; Michalak, J.; Andreadaki, F. J.; Kostas, I. D. Inorg. Chim. Acta. 2008, 361, 1562.

13. Welton, T. Chem. Rev. 1999, 99, 2071.

14. Wasserscheid, P.; Keim, W. Angew. Chem. Int. Ed. 2000, 39, 3772.

15. Wilkes, J. S. Green Chem. 2002, 4, 73.

16. Carmicheal, A. J.; Earle, M. J.; Holbrey, J. D.; McCormac, P. B.; Seddon, K. R. Org. Lett. 1999, 1, 997.

17. Xu, L.; Chen, W.; Ross, J.; Xiao, J. Org. Lett. 2001, 3, 295.

18. Vallin, K. S. A.; Emilsson, P.; Larhed, M.; Hallberg, A. J. Org. Chem. 2002, 67, 6243.

19. Park, S. B.; Alper, H. Org. Lett. 2003, 5, 3209.

20. Zhao, D. B.; Fei, Z. F.; Geldbach, T. J.; Scopelliti, R.; Dyson, P. J. J. Am. Chem. Soc. 2004, 126, 15876.

21. Xiao, J. C.; Twamley, B.; Shreeve, J. M. Org. Lett. 2004, 6, 3845.

22. Jin, C. M.; Twamley, B.; Shreeve, J. M. Organometallics 2005, 24, 3020.

23. Luo, S.; Mi, X.; Zhang, L.; Liu, S.; Xu, H.; Cheng, J. P. Angew. Chem. Int. Ed. 2006, 45, 3093.

24. Fukumoto, K.; Yoshizawa, M.; Ohno, H. J. Am. Chem. Soc. 2005, 127, 2398.

25. Yao, Q.; Zhang, Y. Angew. Chem. Int. Ed. 2003, 42, 3395.

26. Wang, L.; Li, H. j.; Li, P. Tetrahedron 2009, 65, 364.

27. Li, B.; Li, Y. Q.; Zheng, W. J.; Zhou, M. Y. Arkivoc 2009, (xi), 165. 